\title{
Novel Treatment Setup for Urethral Cancer: Use of the Prone Technique for Radiotherapy of the Penis
}

\author{
Aurash Naser-Tavakolian ${ }^{\mathrm{a}} \quad$ Cindy $\operatorname{Tran}^{\mathrm{b}} \quad$ Marissa Timoteo \\ Raymond Chiu ${ }^{a}$ Leslie Ballas ${ }^{a}$ \\ aDepartment of Radiation Oncology, Keck School of Medicine of USC, USC Norris \\ Comprehensive Cancer Center, Los Angeles, CA, USA; ${ }^{b}$ Michigan State University College \\ of Human Medicine, East Lansing, MI, USA
}

\section{Keywords}

Urethral cancer - Radiotherapy · Prone technique

\begin{abstract}
Urethral cancer (UC) is a rare malignancy with a poor prognosis. Since local recurrence is common and associated with morbidity, case series have reported on the use of adjuvant radiotherapy. Radiotherapy treatment setup for malignancies of the penis can be challenging because of variability in anatomic positioning. This variability can lead to lack of reproducibility. We propose a novel external beam radiotherapy technique for the treatment of UC: prone positioning. This technique has been used to treat breast cancers successfully and can be used to treat any variety of penile malignancies. We present 2 patients who were treated using this positioning.




\section{Introduction}

Urethral cancer (UC) is a rare malignancy with a poor prognosis [1,2]. Treatment of UC is individualized, as there are no prospective trials to provide recommendations given the rarity of urinary tract malignancies. Since local recurrence is common and associated with morbidity and decreased disease-specific survival, adjuvant radiotherapy has been studied in limited case series $[3,4]$.

Treatment setup for urethral radiotherapy in men is challenging as the positioning of male anatomy is subject to variability, thereby raising concerns for radiation toxicity in normal adjacent tissues and reduced treatment precision. Radiotherapy for UC is based on the technique for penile cancer radiotherapy. For external beam radiation therapy (EBRT), the patient is positioned supine on a treatment couch with the penis encased vertically in a wax block for immobilization. Because of variability in penis position and treatment-related edema, the supine technique can lack reproducibility [5].

We propose a novel EBRT treatment setup for UC that features the prone technique to treat the penis, bypassing the need for immobilization devices used in the supine technique. Two cases are presented of men with urethral squamous cell carcinoma (SCC) who underwent adjuvant radiotherapy while prone. This report illustrates the design, reproducibility, and applications of the prone technique for administering EBRT to the penis.

\section{Case Presentations}

Case A

A 51-year-old male with a history of urethral strictures since the age of 20 presented with urethral obstruction and inability to urinate. The patient underwent cystoscopy which detected granulation tissue concerning for carcinoma. A suprapubic catheter was placed and a biopsy showed invasive well-differentiated SCC. Staging computed tomography (CT) of the chest, abdomen, and pelvis revealed no evidence of metastatic disease. The patient received neoadjuvant chemotherapy with 4 cycles of ifosfamide, paclitaxel, and cisplatin (ITP). He subsequently underwent urethrectomy, urethroplasty, and perineal urethrostomy with pathology confirming urethral SCC (stage III, pT3cN0) measuring $3.1 \times 5.1 \mathrm{~cm}$ with extensive involvement of the corpus cavernosum and invasion into skeletal muscle. The radial margin was positive while the proximal and distal margins were free of malignancy. Following surgery, he returned to normal activity without urinary symptoms but he did report penile numbness and erectile dysfunction. The patient was dispositioned to 5,940 cGy in 33 fractions of adjuvant radiotherapy (44 elapsed days) with the prone technique targeting the prior prostatic and penile urethra to decrease local recurrence secondary to the positive radial margin. Eleven months following radiation treatment, the patient developed lung metastases and was treated with chemotherapy. He developed pelvic recurrence approximately 3 years after initial radiotherapy.

Case B

A 56-year-old male with a history of Alport syndrome, 3 renal transplants, chronic obstructive pulmonary disease, and recurrent diffuse urethral stricturing presented with pro- 


\section{Case Reports in Oncology}

Case Rep Oncol 2018;11:268-275

DOI: $10.1159 / 000488935$

(c) 2018 The Author(s). Published by S. Karger AG, Basel www.karger.com/cro

Naser-Tavakolian et al.: Novel Treatment Setup for Urethral Cancer: Use of the Prone Technique for Radiotherapy of the Penis

gressive urethral tightening. The patient had multiple prior urethral reconstructions for hypospadias with his first urethroplasty about 30 years ago followed by multiple urethroplasties and dilations. He had a persistent urethral diverticulum at the penoscrotal junction and his most recent cystoscopy revealed a calcification partially within the wall of the diverticulum. Four months later, he underwent excision of the calcification and reconstruction with urethrostomy. The biopsy identified moderately differentiated urethral SCC (stage III, ypT3N0) extending into adipose tissue and exhibiting focal perineural invasion. Surgical margins were positive. CT of the chest, abdomen, and pelvis 1 month later showed no evidence of recurrent or metastatic disease. Since surgery, the patient experienced spraying and dribbling during urination but otherwise felt well. Two months after resection, he proceeded with adjuvant chemoradiotherapy. The patient was recommended low-dose weekly cisplatin therapy; however, he received only 2 doses concurrent with radiotherapy because of his transplanted kidney function. The patient was dispositioned to 5,940 cGy in 33 fractions of adjuvant radiotherapy with the prone technique targeting the area of resected urethra and proximal urethrostomy to decrease local recurrence secondary to the positive surgical margins. The patient had no evidence of residual, recurrent, or metastatic disease at reimaging 3 months following radiotherapy.

\section{Positioning for CT Simulation of the Penis}

Patients were placed prone atop 2 rectangular Styrofoam blocks separated by a gap inbetween allowing for gravity to suspend the penis inferiorly into the treatment field (Fig. 1). Patients wore a jock strap with a circumferential ace bandage to secure the testicles from entering the radiation field. No urethral catheter was inserted. CT simulation did not use contrast. Laser alignment marked crosshairs on the patients' skin in a stable position with "BBs" placed over the marks to enable visualization on CT.

\section{D Treatment Planning}

The clinical target volume (CTV) was defined in conjunction with the surgeon as areas at risk for microscopic extension. The CTV in Case A corresponded to the prior prostatic and penile urethra, while Case B targeted the resected urethra and proximal urethrostomy. The planning target volume (PTV) was defined as the CTV plus a $5 \mathrm{~mm}$ expansion in all directions to account for setup error and organ motion. The PTV was then cropped $3 \mathrm{~mm}$ from the skin. No bolus was required.

Parallel opposed beams with 7 fields using field-in-field technique with dynamic wedges were used to maximize the PTV dose and exclude as much of the rectum and bladder as possible (Fig. 2, 3). Dose volume histograms were generated in each case featuring CTV, PTV, bladder, rectum, and more (Fig. 4, 5).

\section{Administering Radiotherapy and Prone Technique Reproducibility}

Each successive fraction involved reproducing the prone setup and aligning the patients' crosshair markings. A diverging light field visually matched the beam to the penis. Pretreatment CT imaging was obtained to ensure the patients were aligned with appropriate precision. The approximate time for setup and patient alignment was 5--10 min. No adjustments were made if alignment fell within $0.2 \mathrm{~cm}$ of the expected position and any positional shifts guided by CT imaging were recorded with each fraction. The mean daily shift data in the 


\section{Case Reports in Oncology}

vertical, longitudinal, and lateral dimensions are presented in Table 1. Flash was used in an effort to reduce the inaccuracy caused by target organ movement and setup error. Patients did not exhibit difficulty with maintaining stability while prone.

\section{Discussion}

The advantages of the prone technique become evident when considering the limitations of the traditional supine technique for radiotherapy delivery to the penis. For the supine technique, a $10 \times 10 \mathrm{~cm}$ block composed of wax or Perspex with an internal cylindrical compartment is standard for immobilizing the penis to provide full-dose EBRT. Using the Perspex block can become increasingly more uncomfortable for patients during the course of treatment and, if not fit snugly, the penis will decline within the chamber leading to underdosing across shorter penile length [5]. Moreover, the greatest benefit of the Perspex block is that it provides full buildup to the skin surface allowing for use of parallel opposed beams. However, the skin is not a target in the treatment of UC, making the bolus effect of Perspex unnecessary.

The prone setup was reproducible within 5--10 min and required minimal adjustments in position. CBCT is required to align patients daily because of penile mobility. Patients found the positioning to be comfortable and maintained stability with ease while prone. Furthermore, utilizing gravity to suspend the penis and restraining the testicles during radiotherapy could potentially decrease the likelihood of exposing normal adjacent tissues. Prone EBRT to the penis has been described using water baths in order to provide adequate dose buildup to the skin, but again, neither the water bath nor bolus is necessary in the treatment of UC [5]. The prone treatment plans were well tolerated and demonstrated outcomes similar to those of supine EBRT for UC.

Use of the prone technique in treating UC with radiotherapy has not been previously described in the literature. Larger studies are warranted to evaluate potential adverse effects associated with EBRT using the prone technique as this report is limited by size. The application of this novel approach to delivering EBRT is not exclusive to UC and can be correlated to other penile malignancies such as sarcoma. In conclusion, the prone technique offers a feasible and innovative way to administer radiotherapy for UC that benefits from its simplicity, cost-effectiveness, and reproducibility.

\section{Statement of Ethics}

The authors have no ethical conflicts to disclose.

\section{Disclosure Statement}

The authors have nothing to disclose. 


\section{Case Reports in Oncology}

\begin{tabular}{l|l} 
Case Rep Oncol 2018;11:268-275 \\
\hline DOI: 10.1159/000488935 & $\begin{array}{l}\text { (c) } 2018 \text { The Author(s). Published by S. Karger AG, Basel } \\
\text { www.karger.com/cro }\end{array}$
\end{tabular}

Naser-Tavakolian et al.: Novel Treatment Setup for Urethral Cancer: Use of the Prone Technique for Radiotherapy of the Penis

\section{Funding Sources}

This case report did not receive financial support.

\section{References}

1 Rabbani F. Prognostic factors in male urethral cancer. Cancer. 2011 Jun;117(11):2426-34.

2 Gakis G, Witjes JA, Compérat E, Cowan NC, De Santis M, Lebret T et al.; European Association of Urology. EAU guidelines on primary urethral carcinoma. Eur Urol. 2013 Nov;64(5):823-30.

3 Dalbagni G, Zhang ZF, Lacombe L, Herr HW. Male urethral carcinoma: analysis of treatment outcome. Urology. 1999 Jun;53(6):1126-32.

4 Narayan P, Konety B. Surgical treatment of female urethral carcinoma. Urol Clin North Am. 1992 May;19(2):373-82.

5 Crook J, Ma C, Grimard L. Radiation therapy in the management of the primary penile tumor: an update. World J Urol. 2009 Apr;27(2):189-96.

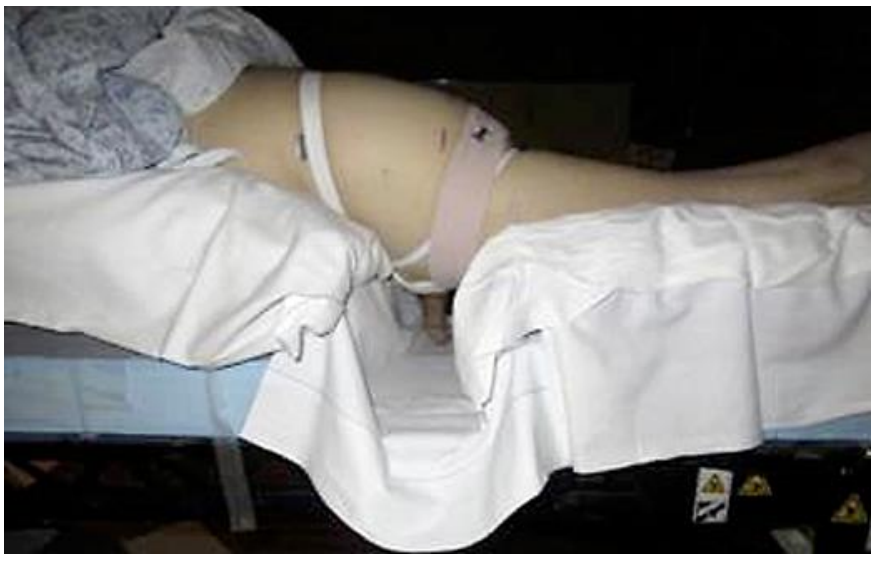

Fig. 1. Prone position setup. 


\section{Case Reports in Oncology}

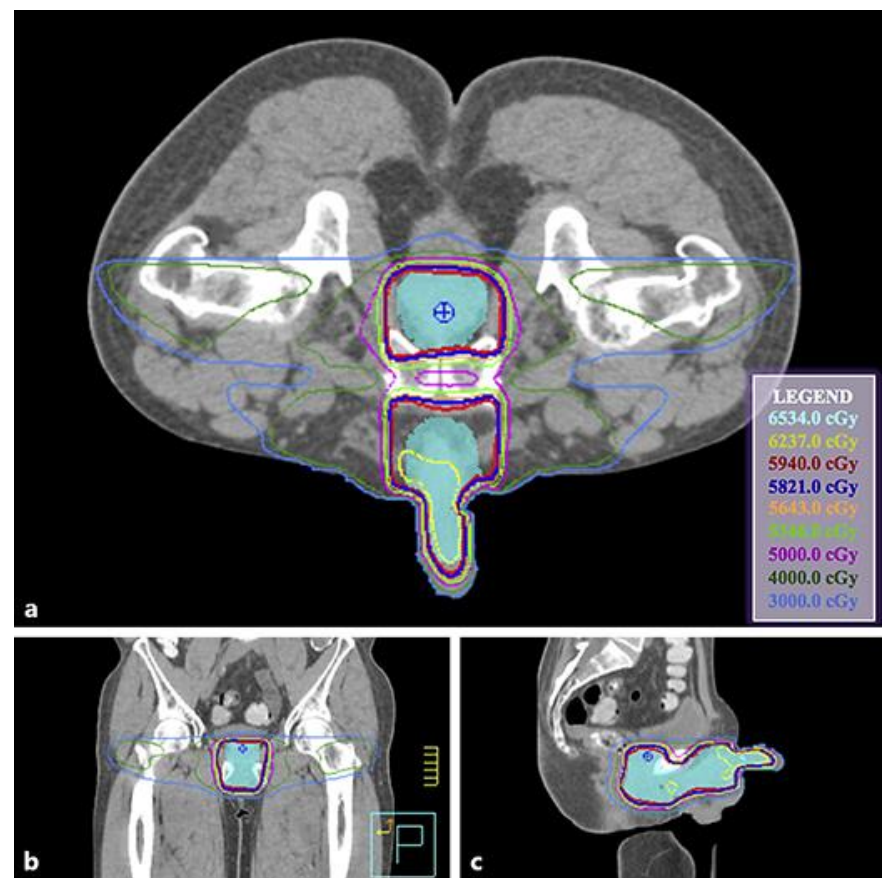

(c) 2018 The Author(s). Published by S. Karger AG, Basel www.karger.com/cro

Naser-Tavakolian et al.: Novel Treatment Setup for Urethral Cancer: Use of the Prone Technique for Radiotherapy of the Penis

Fig. 2. Transaxial (a), coronal (b), and sagittal (c) views of the treatment plan in Case A. PTV is illustrated in cyan. 


\section{Case Reports in Oncology}

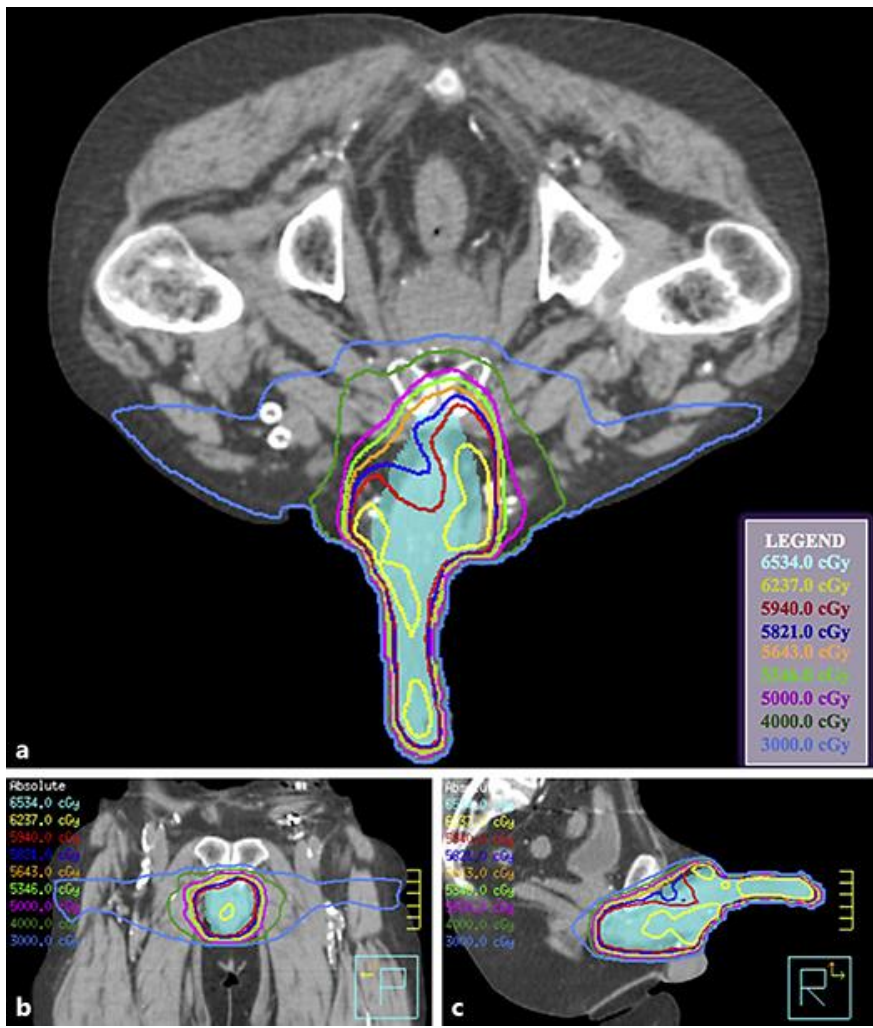

Fig. 3. Transaxial (a), coronal (b), and sagittal (c) views of the treatment plan in Case B. PTV is illustrated in cyan.

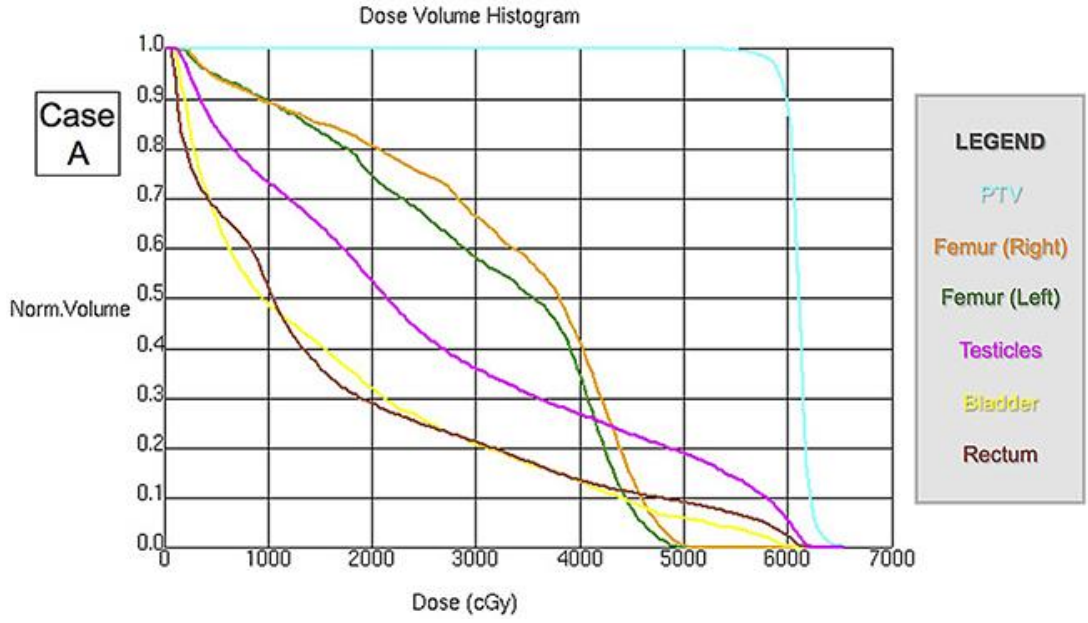

Fig. 4. Dose volume histogram for Case A.

Naser-Tavakolian et al.: Novel Treatment Setup for Urethral Cancer: Use of the Prone Technique for Radiotherapy of the Penis 


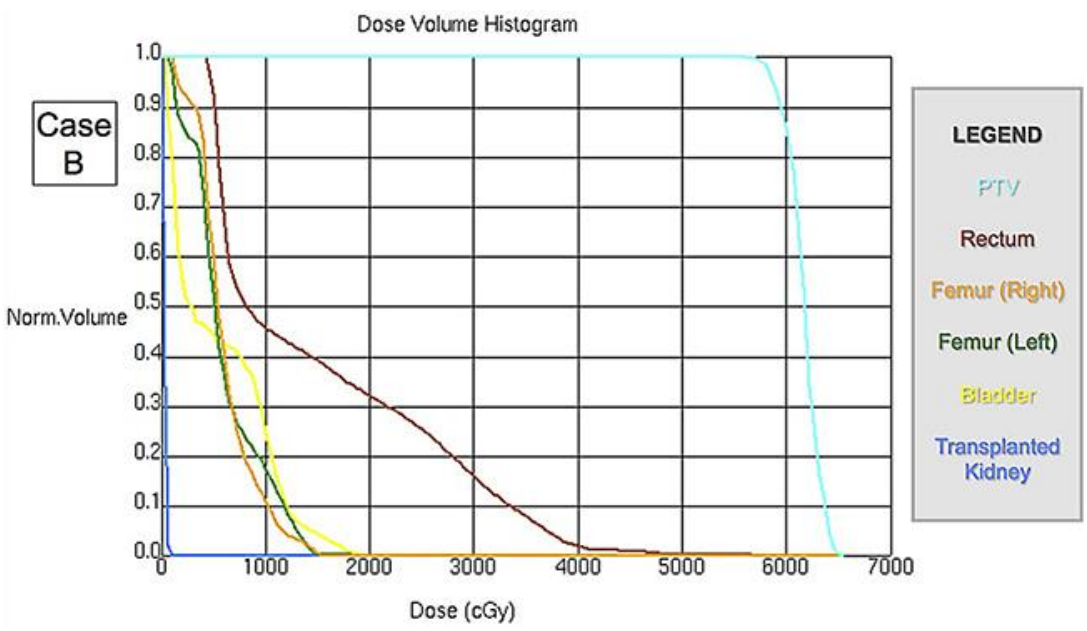

Fig. 5. Dose volume histogram for Case B.

Naser-Tavakolian et al: Novel Treatment Setup for Urethral Cancer: Use of the Prone Technique for Radiotherapy of the Penis

Table 1. Mean daily shift data relative to initial marking on EBRT fraction 1 for urethral cancer while prone

\begin{tabular}{clll}
\hline & \multicolumn{2}{c}{ Directional shift per fraction, cm } \\
\cline { 2 - 4 } & vertical & longitudinal & lateral \\
\hline Case A & & & \\
Mean & 0.38 & 0.14 & 0.28 \\
Median & 0.4 & 0.2 & 0.3 \\
Case B & & & \\
Mean & 1.3 & 1.0 & 0.9 \\
Median & 1.3 & 1.0 & 0.8 \\
\hline
\end{tabular}

